Portland State University

PDXScholar

$5-7-1991$

\title{
An Analysis of the $\mathrm{pH}$ Tolerance and Substrate Preference of Isolated Skeletal Muscle Mitochondria from Bufo marinus and Rana catesbeiana
}

Jeffrey Mark Duerr

Portland State University

Follow this and additional works at: https://pdxscholar.library.pdx.edu/open_access_etds

Part of the Biology Commons

Let us know how access to this document benefits you.

\section{Recommended Citation}

Duerr, Jeffrey Mark, "An Analysis of the pH Tolerance and Substrate Preference of Isolated Skeletal Muscle Mitochondria from Bufo marinus and Rana catesbeiana" (1991). Dissertations and Theses. Paper 4201.

https://doi.org/10.15760/etd.6085

This Thesis is brought to you for free and open access. It has been accepted for inclusion in Dissertations and Theses by an authorized administrator of PDXScholar. Please contact us if we can make this document more accessible: pdxscholar@pdx.edu. 
AN ABSTRACT OF THE THESIS OF Jeffrey Mark Duerr for the Master of Science in Biology presented May 7, 1991.

Title: An Analysis of the $\mathrm{pH}$ Tolerance and Substrate Preference of Isolated Skeletal Muscle Mitochondria from Bufo marinus and Rana catesbeiana.

APPROVED BY THE MEMBERS OF THE THESIS COMMITTEE:

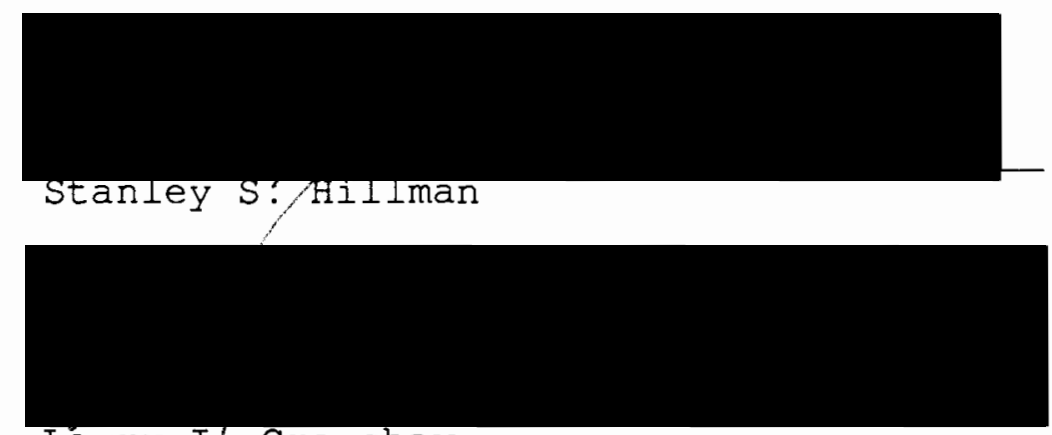
Larry I\% Crawshaw
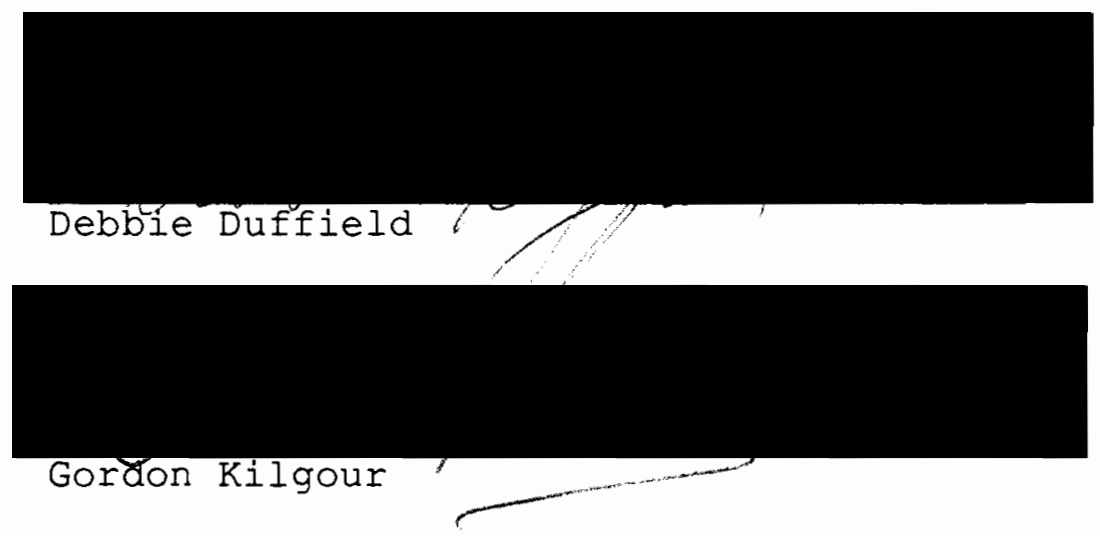

The concept of maximal oxygen consumption (VO2 max) implies that there is a limit to the amount of oxygen that can be consumed by an organism in a given time. Many attempts have been made to delineate exactly what factor or factors are limiting to $\mathrm{VO}_{2} \max$. Current models focus on the 
steps over which oxygen must traverse in order to get from the ambient air to the mitochondria. However, no model has addressed the issue of whether or not carbon dioxide flux may impose a limit to $\mathrm{VO}_{2}$ max. Impedance of carbon dioxide flux may have a deleterious effect on intracellular $\mathrm{pH}$, which in turn, may effect mitochondrial oxidative performance. Experimentally controlled in vitro respiration of isolated skeletal muscle mitochondria can reflect in vivo performance under similar conditions. Skeletal muscle mitochondria were isolated from the "aerobic" toad Bufo marinus and the "anaerobic" frog Rana catesbeiana. Oxidative capacity, or $\mathrm{VO}_{2}$ max, was evaluated for mitochondria subjected to varying physiological pH ranging from 6.1-7.4. Another aspect of mitochondrial metabolism involves muscle fibre types. In several vertebrates, muscle fibres are specialized for either aerobically or anaerobically driven locomotion, and as a metabolic consequence utilize different sets of substrates. Skeletal muscle mitochondria from $\underline{B}$. marinus and $\underline{R}$. catesbeiana were allowed to respire in media containing either pyruvate, alpha-ketoglutarate, oxaloacetate, beta-hydroxybutyrate, or octanoyl-carnitine in order to assess whether or not mitochondria from amphibians with different locomotory patterns preferred different substrates.

For both species of amphibian, mitochondrial oxygen consumption was maximal at a pH range of $7.3-7.6$ and declined 
significantly $(\mathrm{p}<0.05)$ at all $\mathrm{pH}<7.3$. Respiratory control ratio ( $R C R$ ) values decreased significantly at all $\mathrm{pH}<6.9$. It therefore appears that mitochondria indeed have a $\mathrm{pH}$ optimum (within physiological ranges experienced) and that $\mathrm{CO}_{2}$ flux is a factor that may place limits on $\mathrm{VO}_{2}$ max.

Substrate preference was identical for both species of amphibian corroborating studies which contend both genera of amphibians have mixed skeletal muscle fibre types. Interspecific comparisons revealed that $B$. marinus had a significantly higher $\mathrm{VO}_{2}$ max in four of the five substrates. This augmented aerobic performance sustains the locomotory metabolic requisites terrestrial excursions demand. 
AN ANALYSIS OF THE pH TOLERANCE AND SUBSTRATE PREFERENCE OF ISOLATED SKELETAL MUSCIE MITOCHONDRIA FROM BUFO MARINUS AND RANA CATESBEIANA

by

JEFEREY MARK DUERR

A thesis submitted in partial fulfillment of the requirements for the degree of

\author{
MASTER OF SCIENCE \\ in \\ BIOLOGY
}

Portland State Universtity

1991 
TO THE OFFICE OF GRADUATE STUDIES:

The members of the Committee approve the thesis of Jeffrey Mark Duerr presented May 7, 1991.

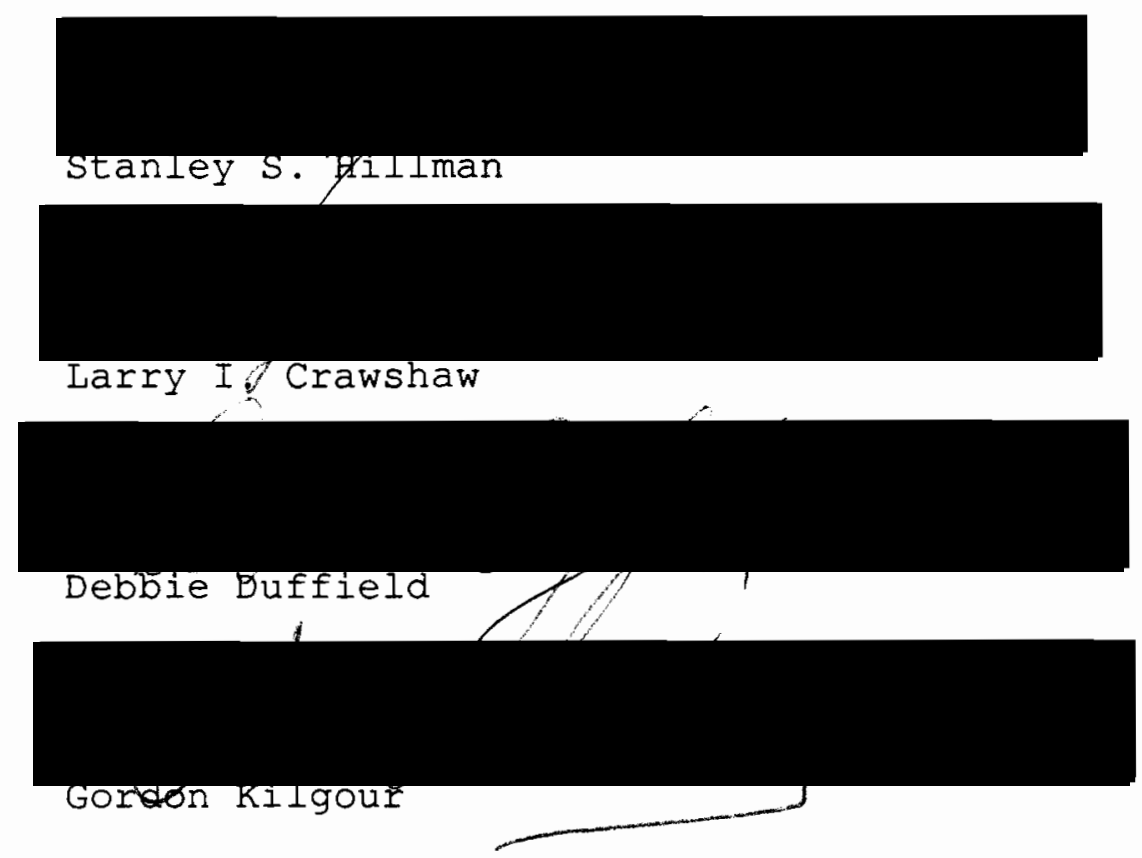

APPROVED :

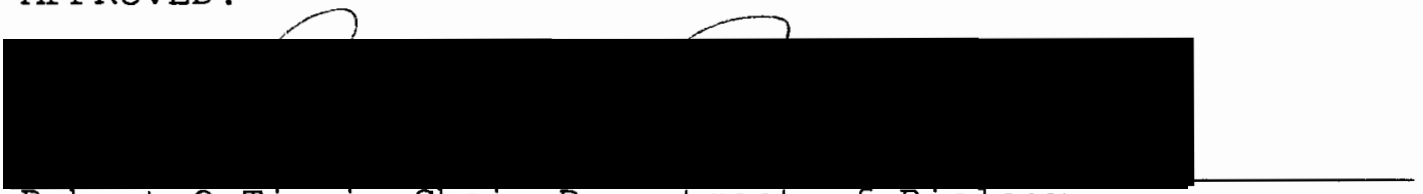

Robert O. Iinnin, Chair, Department of Biology

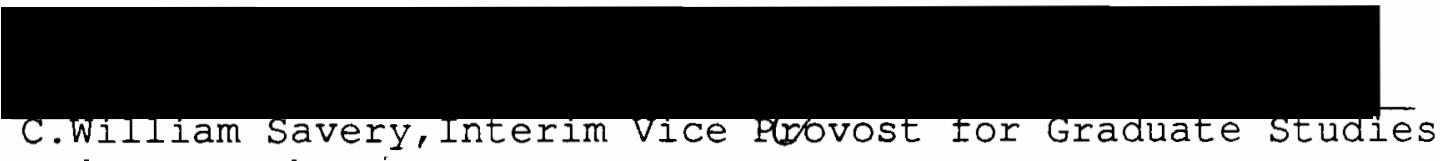
and Research 


\section{ACKNOWLEDGEMENTS}

The dedication to the quality of science and academics, the energy, and the enthusiasm of many individuals influenced the final completion of this work. I wish to thank all the members of my committee, and in particular, I would like to thank my advisor Stan Hillman for his excellent training and relaxed encouragement.

Certainly my progress would have been greatly impeded if not for the unending emotional and financial (mainly for my Rabbit) support from both my parents and family in Spokane. No value could be placed on the guidance and teaching received over coffee at the Ramey's farm in Hillsboro, and of course, the campanionship and genuine friendship I shared with sandy. I also could not forget all the late-night phone calls to Dave in seattle concerning school, life, jazz, etc...

Finally, I dedicate this work to God, with the following intention, best summarized by Sir Thomas Browne: "The Wisdom of God receives small honour from those vulgar Heads that rudely stare about, and with a gross rusticity admire His works: those highly magnifie Him, whose judicious inquiry into His acts, and deliberate research into His Creatures, return the duty of a devout and learned admiration... Nature is the Art of God." 
TABLE OE CONTENTS

PAGE

ACKNOWLEDGEMENTS ...............................

IIST OF EIGURES $\ldots \ldots \ldots \ldots \ldots \ldots \ldots \ldots \ldots \ldots \ldots$

INTRODUCTION .......................... 1

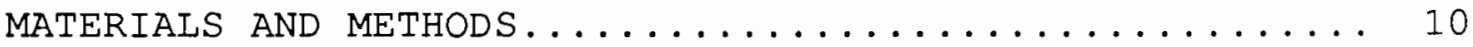

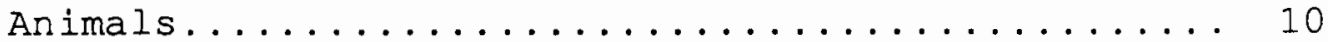

Isolation of Mitochondria................ 10

Measurement of Respiration............... 11

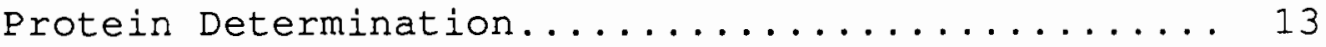

Statistical Comparisons................. 13

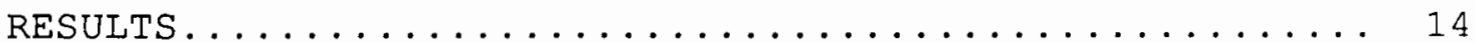

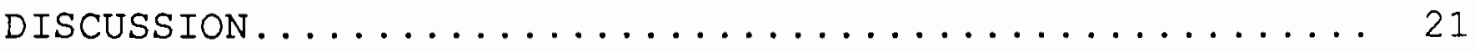

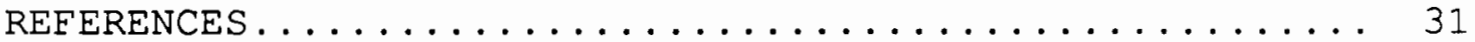




\section{IIST OF FIGURES}

FIGURE

PAGE

1. The effect of $\mathrm{pH}$ on state 3 respiration and state 4 respiration in isolated skeletal muscle mitochondria from both anuran species............. 15

2. The effect of $\mathrm{pH}$ on respiratory control ratios in isolated skeletal msucle mitochondria form Bufo marinus and

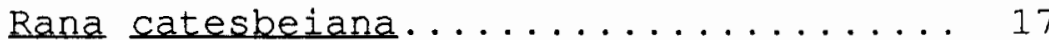

3. State 3 substrate preference in isolated skeletal muscle mitochondria........ 19

4. State 4 substrate preference of isolated skeletal muscle mitochondria......... 21

5. RCR substrate preference of isolated skeletal muscle mitochondria............... 23 


\section{INTRODUCTION}

There are four series steps in oxygen exchange between the air and the cells. Any one or combination of these steps may in some way contribute the major resistance to flux, which would thus ultimately place limits on the volume of oxygen that may be maximally consumed ( $\mathrm{VO}_{2}$ max) by an organism. Initially, there is pulmonary convective $\mathrm{O}_{2}$ exchange which depends on both the lung ventilation rate and the $\mathrm{PO}_{2}$ (partial pressure of oxygen) difference between the lung and the ambient air. Second, factors including lung surface area, degree of vascularity, vessel wall thickness, and lung-arterial $\mathrm{PO}_{2}$ difference, may dictate the rate of pulmonary diffusive exchange. Cardiovascular convective exchange is the third step in oxygen transport. Transport here is dependent upon systemic mass blood flow and arteriovenous differences in oxygen concentration. Finally, tissue diffusive exchange is determined by capillary surface area, path length from capillaries to mitochondria, and $\mathrm{PO}_{2}$ difference between capillary blood and cells. The number and volume of mitochondria could also potentially set limits to $\mathrm{VO}_{2} \max$.

Taylor and Weibel (1981) have hypothesized that the entire respiratory system is designed in such a way that each structural element matches the functional needs for maximal 
oxygen flux at each step. However, in experiments where respiratory variables were scaled to animals of different masses, correlations could not be made with scaled $\mathrm{VO}_{2}$ max values (Garland and Huey, 1987). Thus, the symmorphosis proposed by Taylor and Weibel (1981) is subject to challenge. Recent experimental focus has been on two of four rate limiting steps: cardiovascular and mitochondrial. Evidence exists for both possibilities.

Data that support mitochondria as a source for an aerobic limit include studies correlating $\mathrm{VO}_{2}$ max with the number or density of mitochondria (Hoppeler 1990; Hoppeler et al. 1987). Prior to these results, evidence in support of mitochondria as the limiting factor in $\mathrm{O}_{2}$ flux was based upon correlations in anuran amphibians between maximal aerobic capacity and levels of citrate synthase, a TCA cycle enzyme, and consequently a mitochondrial marker (Putnam and Bennett, 1983; Cummings 1979).

Recently, a model has been developed for maximal $\mathrm{O}_{2}$ transport in anuran amphibians (Withers and Hillman, 1988). In this model, maximal resistance to $\mathrm{O}_{2}$ flux exists at the level of systemic $\mathrm{O}_{2}$ transport. Accordingly, increases in aerobic capacity provided by increases in cardiovascular $\mathrm{O}_{2}$ transport are concommitant.

Data exist which corroborate this model of a cardiovascular limit to $\mathrm{O}_{2}$ flux. For example, regression analysis of ventricular mass and hemoglobin concentration in 
Rana catesbeiana revealed that these two factors accounted for significant fractions of the variation in $\mathrm{VO}_{2}$ max (Hillman, 1976). Experiments with anuran amphibians involving decreases in heart rate and experimentally-induced anemia produced a marked decline in $\mathrm{VO}_{2}$ max (Hillman; 1981, 1980).

Pietro diprampero (1990) has developed a multifactorial model for oxygen flux in humans, derived from the $\mathrm{O}_{2}$ conductance equation. This model delineates those factors which may place a limit on $\mathrm{VO}_{2}$ max. According to this model, the limits are shared between systemic $\mathrm{O}_{2}$ transport, responsible for approximately $70 \%$ of the resistance, and a peripheral factor reponsible for the remaining $30 \%$ of resistance.

All hypotheses to date have considered only oxygen as a potential limit to $\mathrm{VO}_{2} \max$. However, active uptake of oxygen by mitochondria is concommitant with the production of carbon dioxide. Carbon dioxide efflux occurs over the same series of steps (in reverse) as does oxygen and it must be removed at a rate such that metabolic acidosis be avoided. The rate of clearance must therefore balance the rate of oxygen consumption, and, if any of the conducting steps is rate limiting to carbon dioxide flux, oxygen flux may be reduced. An excess amount of carbon dioxide at the cellular level will lower intracellular $\mathrm{pH}$ and could affect mitochondrial function. The potential effects of increased carbon dioxide 
concentration on $\mathrm{VO}_{2}$ max may be tested by ascertaining whether or not mitochondrial aerobic capacity is impaired at any $\mathrm{pH}$ within a physiological range. It should be pointed out that once a pH effect has been confirmed, a more direct test would involve determining whether or not $\mathrm{VO}_{2} \max$ diminishes for the whole organism exercising under increasing partial pressures of $\mathrm{CO}_{2}$.

Oxidative metabolism can be studied in isolated mitochondria that are subjected to experimental variation. The respiratory chain is capable of existing in any one of five respiratory steady states depending on exogenous levels of ADP and/or substrate (Chance and Williams, 1956). Relevant to most research are states 2,3 , and 4 .

According to Chance and Williams (1956), these respiratory states are defined as follows. State 2 is a 'starved' state in which ADP has been exhausted through substrate oxidation and is characterized by zero uptake of $\mathrm{O}_{2}$. State 4 is also distinctively absent of any $\mathrm{O}_{2}$ consumption and represents the exhaustion of added ADP during a period of adequate substrate availability. State 3 is the 'active' state of rapid respiration in which substrate is oxidized, $A D P$ is phosphorylated, and the respiration chain itself is the rate-limiting factor (Byrne and Trounce, 1985). The best measure of the physiological intactness, or structural and functional integrity, of the mitochondria is the quotient of state 3 divided by state 4 , known as the 
respiratory control ratio (RCR). The best measure of optimum conditions for isolated mitochondria however, is the maximum respiratory rate, or the value of state 3 (Lea and Hillman, 1990).

Performance of isolated mitochondria under controlled conditions in vitro can reflect mitochondrial metabolism in vivo. In this way, $\mathrm{VO}_{2} \mathrm{max}$ in isolated mitochondria may be compared to $\mathrm{VO}_{2}$ max in the whole organism, albeit indirectly. Animals with different degrees of tolerance to variables directly affecting $\mathrm{VO}_{2}$ max are useful models for comparison.

Anuran amphibians have varying tolerance to dehydration depending on their degree of terrestriality (Thorson, 1955). By experimentally varying levels of dehydration in the terrestrial toad Bufo marinus and the aquatic frog Rana catesbeiana, it was found both cardiovascular and aerobic capacity were similarly compromised (Hillman, 1987). In addition, this compromise was more rapid in the frog.

These two amphibians with their varying levels of dehydration tolerance are useful models for mitochondrial experimentation. Physiological variables such as osmotic pressure can be experimentally varied in isolated mitochondria preparations. If values within normal physiological variation are utilized, it may be determined whether or not mitochondiral $\mathrm{VO}_{2} \max$ is affected.

Isolated skeletal muscle mitochondria obtained from Bufo marinus and Rana catesbeiana were subjected to osmotic 
concentrations ranging from $220 \mathrm{mosm}$ to $420 \mathrm{mosm}$ in $40 \mathrm{mosm}$ increments and revealed no significant differences in $\mathrm{VO}_{2}$ max. Also, $\underline{B}$. marinus maintained a significantly higher oxygen consumption than did $\underline{R}$. catesbeiana (Hillman, Lea, and Duerr, 1991). Over the same range of osmotic concentration, organismal $\mathrm{VO}_{2}$ max decreases, thus, these data corroborate pre-existing data (Hillman, 1987) which do not support a mitochondrial limit to aerobic capacity. The effect of $\mathrm{pH}$ remains to be delineated.

Differences in yield and coupling of isolated mitochondria from $\underline{B}$. marinus and $\underline{R}$. catesbeiana indicate quantitative as well as qualitative differences (Hillman et al. 1991). In anuran amphibians, these differences in both quantity and quality may be correlated with metabolic differences between aerobic, Type I, red muscle fibres and anaerobic, Type II, white muscle fibres. $\mathrm{VO}_{2}$ max has been correlated with the quantity of mitochondria in mammals (Hoppeler et al. 1987).

The oxidative use of a muscle depends on its function (Schmidt-Nielsen, 1984). Toads are primarily terrestrial and rely on slow, consistant Type I muscle activity for locomotion on dry land. Frogs on the other hand are primarily aquatic and use Type II muscle bursts of activity to escape from predation. Although toads and frogs have mixed locomotory muscle fibre types (Putnam and Bennett, 1983), each animal's relative behavior and level of aerobic 
performance indicates predominating red or white fibre types.

Swimming speeds of experimental sprint-trained Rainbow trout, compared with untrained control trout, demonstrated an increased level of both performance and glycolytic enzyme activity. Since Rainbow trout body musculature is $90 \%$ white muscle, level of Type II or white muscle fibre performance was correlated with increased glycolytic enzyme activity. These data offer additional support for correlations between muscle fibre type and activity (Pearson, Spriet, and Stevens, 1990).

Electrophysiological studies indicate that red muscle fibres are specialized for long-term, low intensity exercise while white muscle fibres are involved in burst, high intensity exercise (Rayner and Keenan, 1967). Long-term exercise by red muscle is fueled by the mitochondrial oxidation of pyruvate, derived from lactate, glycolysis, or some amino acids, as well as fatty acids, and ketone bodies. Aerobic processes are indeed more effecient than anaerobic: each molecule of glucose yields 36 molecules of ATP aerobically, whereas anaerobic mechanisms yield 2 molecules of ATP per glucosyl unit (Stryer, 1988). Burst-type exercise requires ATP at a rate higher than that which may be met by mitochondrial oxidative phosphorylation. As a consequence, a non-oxidatative anaerobic glycolytic pathway is employed by 
white muscle, which net only 2 molecules ATP per molecule glucose, but at a markedly higher rate (Stryer, 1988).

Substrate utilization by mitochondria in vitro reflect the possible array of molecules oxidized in vivo. Studies on the oxidative properties of carp muscle (Moyes et al. 1989; Johnston, 1977) suggest that mitochondria from different fibre types are specialized for oxidation of different sets of substrates as reflected by their substrate preference. Currently, it is believed these differences are a consequence of differing enzyme activities and types of transport shuttles (Person et al. 1990; Moyes et al. 1989, Saier, 1987; Hooker and Baldwin, 1979; Johnston, 1977).

No data is as yet published for amphibian substrate preference and the following substrates are a comparative list commonly employed in other vertebrate mitochondrial assays (Moyes et al. 1990). Pyruvate consumption is generally high in most tissues (Moyes et al. 1990) and would be expected to be preferred in tissues that reach anaerobic conditions and must rely on glycolysis for ATP. Alphaketoglutarate consumption may indicate a high protien diet for aerobic tissues as this molecule is the product of transamination reactions for many amino acids and is a Kreb's cycle intermediate. Oxidation of octanoyl-carnitine yields evidence for fatty acid availability, and betahydroxybutyrate is commonly used by vertebrates as fuel during starvation (Moyes et al. 1990). Oxaloacetate is 
considered not to be permeable to most vertebrate mitochondria (Tzagoloff, 1982), a generalization yet to be confirmed for amphibians.

The following study delineates a $\mathrm{pH}$ profile for both Bufo marinus and Rana catesbeiana. Isolated skeletal muscle mitochondria performance is evaluated over a $\mathrm{pH}$ range of 6.1 - 7.6. This range encompasses normal physiological extremes based on blood pH measured from amphibians at rest and following exhaustive exercise (Putnam, 1979). If mitochondrial oxygen consumption diminishes with decreasing $\mathrm{pH}$, then the possibility of carbon dioxide efflux directly affecting oxygen influx is supported. In addition, substrate preferance of isolated skeletal muscle mitochondria from both the toad and frog is assayed and compared. 


\section{MATERIALS AND METHODS}

\section{ANIMALS}

Bufo marinus were purchased from a commercial supplier while Rana catesbeiana were captured from local wild populations in Washington county, Oregon. Both species were maintained in the laboratory and given free access to water. Experiments were performed within three weeks of arrival for toads and two weeks of capture for frog.

\section{ISOLATION OF MITOCHONDRIA}

Animals were doubly pithed and the thigh musculature quickly excised with ice cold scissors. Approximately $5 \mathrm{~g}$ of muscle was weighed in a beaker containing an isolation medium consisting of $0.170 \mathrm{M}$ mannitol, $0.055 \mathrm{M}$ sucrose, $0.005 \mathrm{M}$ EGTA (ethyleneglycol-bis- $\beta$-aminoethyl ether), $0.02 \mathrm{M}$ Tris (2amino-2-hydroxymethyl-1,3-propanediol), 0.5\% bovine serum albumin (BSA) and 50 units/mL of heparin. The final osmotic pressure of 270 mosm was verified with a vapor pressure osmometer (Wescor Inc. Model 523A). Muscle tissue was manually minced with scissors and placed in a bladed homogenizer for approximately 30 seconds at $1000 \mathrm{rpm}$. The homogenate was forced by a threaded piston through $0.8 \mathrm{~mm}$ diameter holes in a custom fabricated tissue press, placed into a Potter-type glass homogenizer (Tri-R Instruments) and 
ground with the first two (toad) or all three (frog) pestles of increasing diameter: $0.15 \mathrm{~mm}, 0.05 \mathrm{~mm}$, and $0.025 \mathrm{~mm}$, at 200 rpm.

The resulting homogenate was spun at $755 \mathrm{x} g$ for five minutes to seperate large cellular debris from the mitochondria, which remained in the supernate. The preserved supernatant was spun for nine minutes at $9800 \mathrm{x} g$ to pellet mitochondria. The pellet from the second spin was resuspended in isolation medium and centrifuged a third time (to yield a more pure mitochondrial isolation) for seven minutes at $9800 \times \mathrm{g}$. The final resuspension involved $200 \mu \mathrm{L}$ of isolation medium with BSA being gently mixed into the second pellet with a glass stirring rod forming a mitochondrial slurry. A $50 \mu \mathrm{L}$ (toad) or $20 \mu \mathrm{L}$ (frog) aliquot was frozen for protein determination.

\section{MEASUREMENT OF RESPIRATION}

Oxygen uptake was measured at 20 degrees Celsius with a Transidyne General Chemical Microsensor Model 1251 calibration cell. Attached to the $0.9 \mathrm{~mL}$ reaction chamber was a oxygen microelectrode, which utilized the polarographic principle to measure oxygen tension within the chamber to be made (See Estabrook, 1967). The voltage of the system was recorded on a strip-chart recorder.

For all assays, the reaction chamber was filled with $870 \mu \mathrm{L}$ of an oxygen uptake medium which consisted of $55 \mathrm{mM}$ 
mannitol, $24 \mathrm{mM}$ sucrose, $10 \mathrm{mM}$ Tris ( $\mathrm{pH}=7.2$ at room temp.), $10 \mathrm{mM} \mathrm{KH} 2 \mathrm{PO} 4,90 \mathrm{mM} \mathrm{KCl}$, and $50 \mathrm{mM}$ malic acid (osmotic pressure $=283$ mosm) . In addition to oxygen uptake media, $10 \mu \mathrm{L}$ of mitochondrial suspension, $10 \mu \mathrm{L}$ of a substrate, and finally, to initiate maximal oxidative activity, $4 \mu \mathrm{L}$ of 100 MM ADP were added.

For assays involving $\mathrm{pH}$ effects, the $\mathrm{pH}$ of the oxygen uptake medium was adjusted to $6.1,6.5,6.9,7.3$, or 7.6 at room temperature with either $\mathrm{HCl}$ or $\mathrm{NaOH}$ and $10 \mu \mathrm{L}$ of $1 \mathrm{M}$ pyruvate was then added as a substrate. For assays involving substrate preference, the $\mathrm{pH}$ of the oxygen uptake medium was held constant at 7.2 and $10 \mu \mathrm{L}(\mathrm{pH}$ adjusted to 7.2 ) of either 1 M pyruvate, 1 M oxaloacetate, 1 M alpha-ketoglutarate, 1 M beta-hydroxybutyrate or $0.2 \mathrm{M}$ octanoyl-DL-carnitine was added. All substrates were obtained from Sigma Chemical Company .

Upon the addition of $0.1 \mathrm{M} A \mathrm{ADP}$, mitochondrial oxidative phosphorylation was traced as the difference in $\mathrm{O}_{2}$ tension within the reaction chamber per minute. This rate of oxygen consumption is considered either state 3 or $\mathrm{VO}_{2} \max$. Respiratory control ratios ( $R C R$ ) were calculated as the ratio of the rate of oxygen uptake in the presence of ADP (state 3) to the rate of oxygen uptake after exhaustion of ADP (state 4 ) or state 3/state 4 (Estabrook 1967, Chance and Williams 1956). 
PROTEIN DETERMINATION

Aliquots of mitochondrial suspensions were thawed, diluted, and added to Folin phenol reagent. Concentration of mitochondrial protein was based on absorbance at $625 \mathrm{~nm}$ after Lowry et al. (1951). Final oxygen consumption was expressed as $\mathrm{nm} \mathrm{O} 2$ consumed per mg mitochondrial protein per minute.

\section{STATISTICAL COMPARISONS}

This experiment was designed to test if and when a significant difference in $\mathrm{VO}_{2}$ max occured at a selected treatment. The data are organized such that there are five treatments replicated three to six times for each anuran. These data sets satisfy the prerequisites for Model I, oneway ANOVA. Significant differences were analyzed with Tukey's test for multiple comparisons (for equal sample size). For unequal sample size, Scheffe's test for multiple comparisons was substituted for Tukey's. A $\mathrm{P}<0.05$ was considered significant.

State 3, state 4, and RCR data for each anuran were analyzed separately using the methods described above. Intraspecific comparisons for data gathered for $\underline{B}$. marinus and $\mathrm{R}$. catesbeiana allowed determination for significant differences within one species. Interspecific comparisons between the toad and frog were performed using independent Student's t-test of each $\mathrm{pH}$ or substrate treatment using any or all three of the respiratory measures. 
RESULTS

For both $\underline{B}$. marinus and $\underline{R}$. catesbeiana state 3 oxygen consumption rates declined significantly $(p<0.05)$ at all $\mathrm{pH}<7.3$ and state 4 values were significantly different at all pH<6.5. Respiratory control ratios decreased significantly at all pH<6.9 for $\underline{B}$. marinus, and for $\underline{R}$. catesbeiana all RCR values were significantly different from each other in the assayed $\mathrm{pH}$ range, Figures 1 and 2 . At $\mathrm{pH}=6.1$, all state 4 values and consequently RCR values are zero for $\underline{B}$. marinus. For $\underline{R}$. catesbeiana, all three respiratory measurements were zero at $\mathrm{pH}=6.1$.

State 3 respiratory rates of the five individual substrates all showed significant differences $(\mathrm{p}<0.05)$ from each other with the exception of pyruvate vs. octanoyl for $\underline{B}$. marinus. Preference of substrate in order of greatest to least is as follows: alpha-ketoglutarate, pyruvate, octanoylcarnitine, beta-hydroxybutyrate, and oxaloacetate, Figure 3.

There were no significant differences among the state 4 substrate oxidation measures, Figure 4. All RCR values for substrate oxidation were significantly different from each other. Substrate preference ranked by RCR values matched that set by state 3 values, Figure 5 .

For $\underline{R}$. catesbeiana, state 3 values were all significantly different with the exception of pyruvate vs. 
octanoyl. The preferred substrate ranking is identical to $\underline{B}$. marinus, Figure 3 .

State 4 data from $\underline{R}$. catesbeiana showed significant differences with the following four exceptions: pyruvate vs. octanoyl, pyruvate vs. beta-hydroxybutyrate, alphaketoglutarate vs. oxaloacetate, and octanoyl vs. betahydroxybutyrate, Figure 4.

Respiratory control ratios were all significantly different from each other in $\underline{R}$. catesbeiana. Substrate preference as determined using $R C R$ values presented the following rank order: octanoyl-carnitine, alphaketoglutarate, pyruvate, beta-hydroxybutyrate, and oxaloacetäe, Figure 5 .

Using Student's t-test, the utilization of each substrate by mitochondria was compared between the two species. Interspecific statistical comparisons made with the five substrates using state 3 data showed that $\underline{B}$. marinus had a significantly $(P<0.05)$ higher $\mathrm{VO}_{2}$ in four of the five substrates. Oxaloacetate was the only substrate that showed no significant difference in utilization between the two species. RCR data revealed no significant differences with any of the substrates, confirming consistant recovery. 
oxygen consumption

(nm oxygen/mg*min)

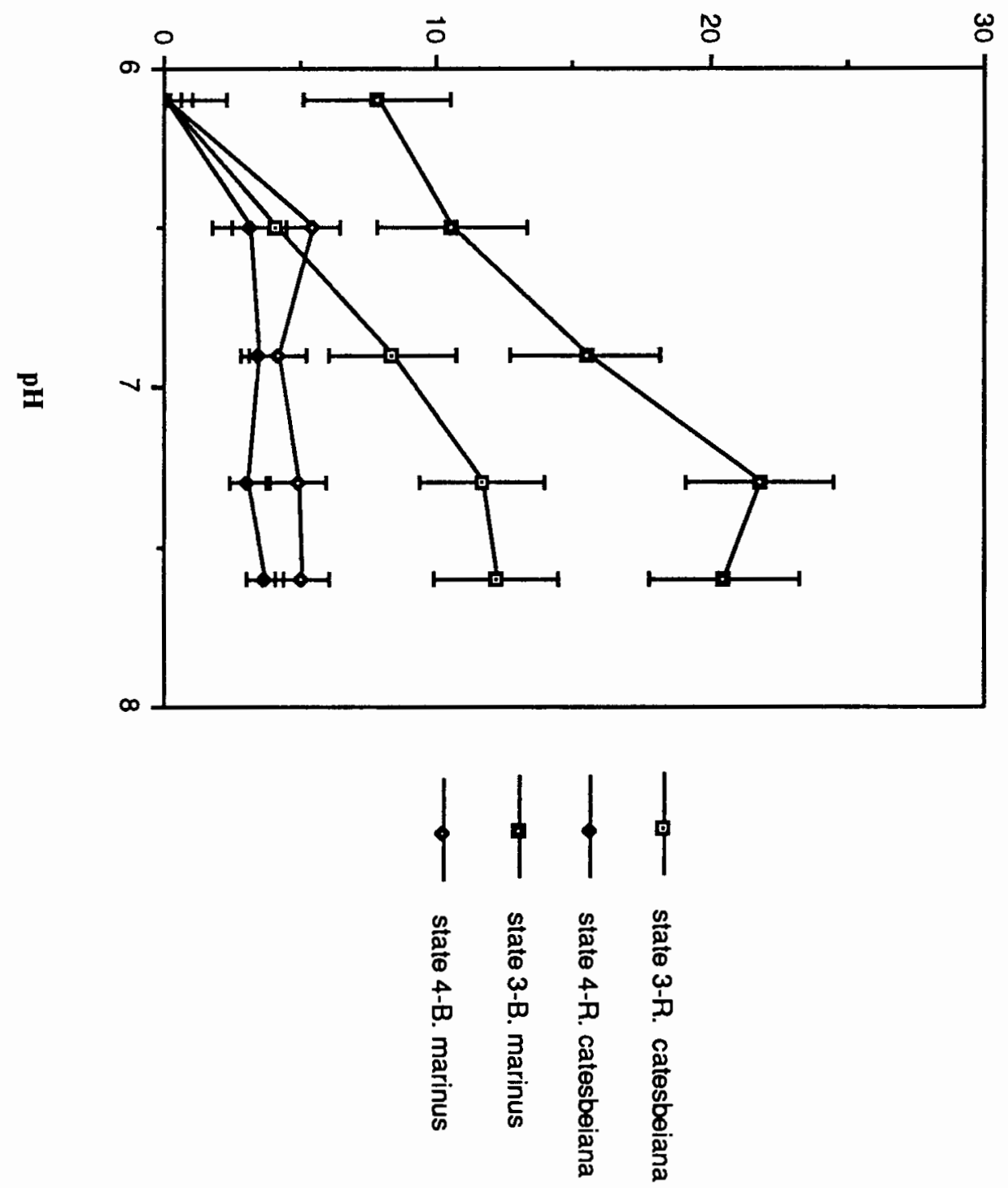

Eigure 1. The effect of $\mathrm{pH}$ on state 3 respiration and state 4 respiration in isolated skeletal muscle mitochondria from both anuran species. Values are mean $+/-$ standard error. 


\section{RCR}

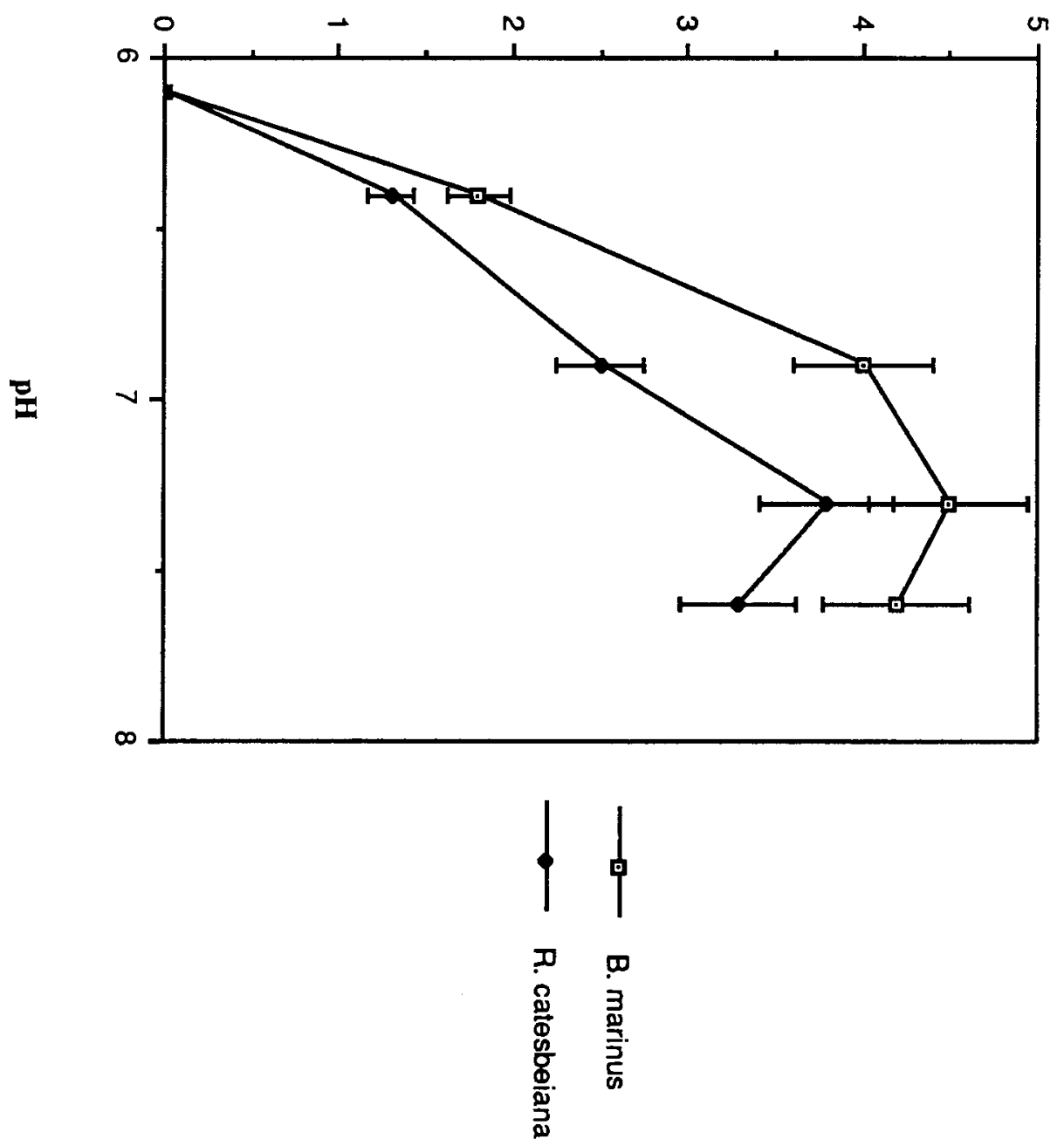

Eigure 2. The effect of $\mathrm{pH}$ on respiratory control ratios in isolated skeletal muscle mitochondria from Bufo marinus and Rana catesbeiana. Values are mean +/- standard error. 


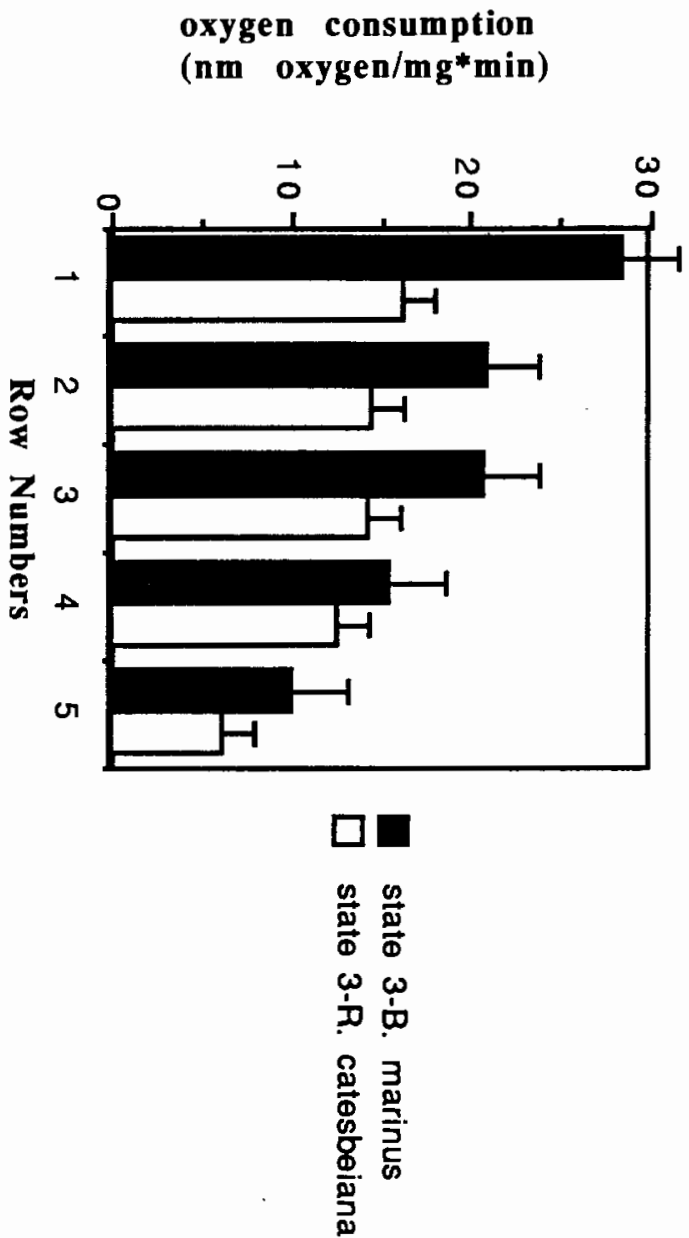

Figure 3 . State 3 substrate preference in isolated skeletal muscle mitochondria. Column 1=alphaketoglutarate, 2=pyruvate, 3=octanoyl, 4=betahydroxybutyrate, $5=0 x a l o a c e t a t e$. Values are mean $+/$ - standard error. 
oxygen consumption

(nm oxygen/mg*min)
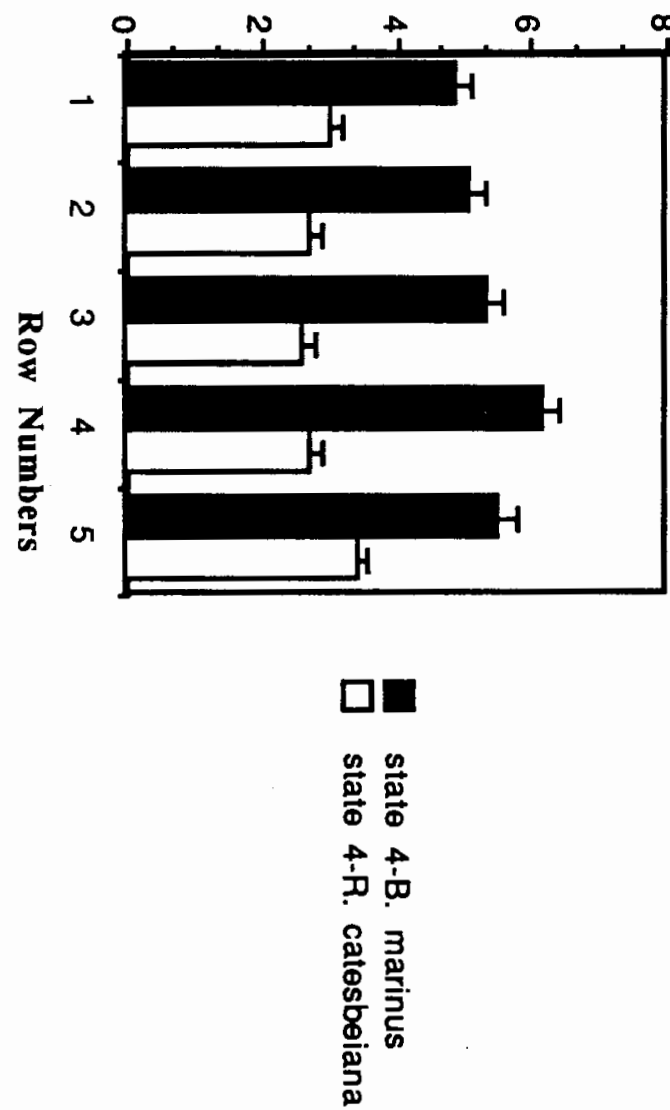

Eigure 4. State 4 substrate preference of isolated skeletal muscle mitochondria. Column 1=alphaketoglutarate, 2=pyruvate, 3=octanoyl, 4=betahydroxybutyrate, 5=oxaloacetate. Values are mean $+/-$ standard error. 


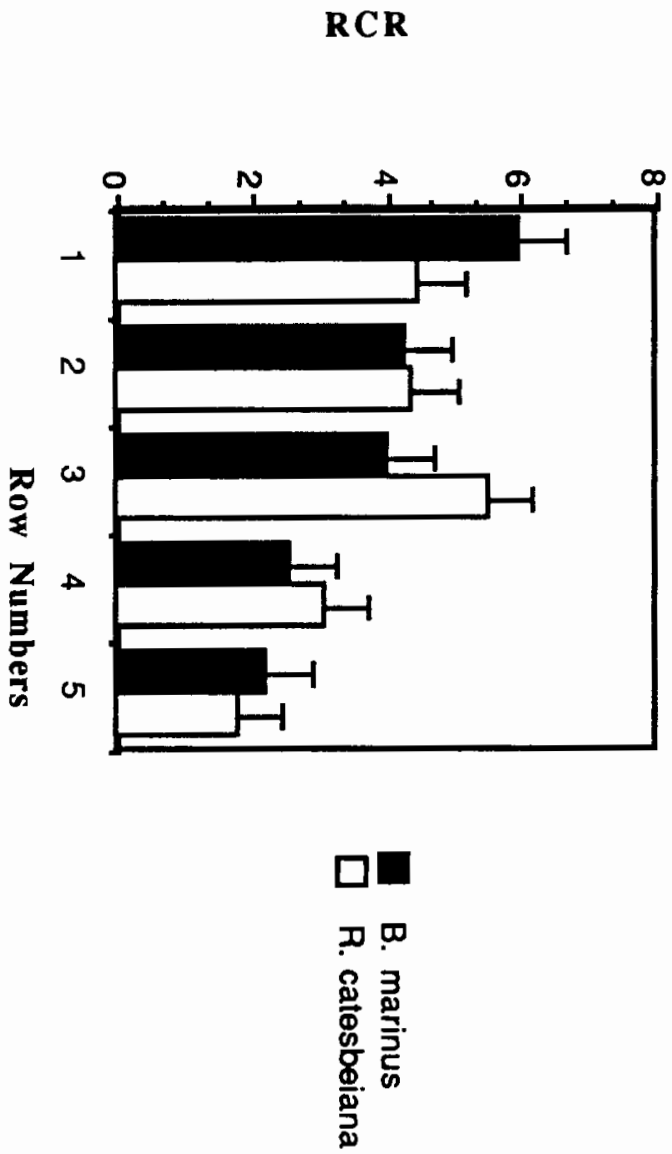

Eigure 5. RCR substrate preference of isolated skeletal muscle mitochondria. Column 1=alphaketoglutarate, 2=pyruvate, 3=octanoyl, 4=betahydroxybutyrate, 5=oxaloacetate. Values are mean= $+/-$ standard error. 


\section{DISCUSSION}

A decrease in $\mathrm{pH}$ ultimately led to a significant $(P<0.05)$ decrease in mitochondrial $\mathrm{VO}_{2}$ max for both species of amphibian. At $\mathrm{pH}<7.3, \mathrm{VO}_{2}$ max declined significantly for both species. RCR values diminished for Bufo marinus at all $\mathrm{pH}<6.9$ and $\mathrm{RCR}$ values at each $\mathrm{pH}$ for Rana catesbeiana were all significantly different.

It is evident that a decrease in intracellular $\mathrm{pH}$, possibly as the result of a decrease in $\mathrm{CO}_{2}$ efflux in vivo, would be detrimental to mitochondrial activity and could therefore place a limit on mitochondrial aerobic capacity and VO2 max. Thus, all potential sources of hydrogen ions must be considered, especially metabolic sources such as $\mathrm{CO}_{2}$ (aerobic) and lactic acid (anaerobic).

During intense anaerobic metabolism, lactic acid accumalation contributes to muscle fatigue (Putnam, 1979). Increases in lactic acid concentration in isolated frog muscle has been correlated with a concurrent decrease in $\mathrm{pH}$. In $\underline{R}$. pipiens, recovery from lactate build-up has been correlated with increased $\mathrm{CO}_{2}$ efflux across the lungs and/or skin, thereby lowering bicarbonate levels in the blood. The ability of the respiratory system to maintain an adequate $\mathrm{CO}_{2}$ efflux seems to be key in maintaining $\mathrm{VO}_{2}$ max potential.

Differences in $\mathrm{pH}$ may effect mitochondrial performance by disturbing the tertiary and/or quaternary structure of 
proteins (Stryer, 1988) and thus affecting carrier protein function. Particular transport processes are governed by proton binding sites on the carriers (LaNoue and Schoolwerth, 1979). The uptake of anions is frequently stimulated by intermembrane space $\mathrm{H}^{+}$ions and efflux of anions such as pyruvate and glutamate may be inhibited by $\mathrm{H}^{+}$(LaNoue and Schoolwerth, 1979). Pyruvate, for example, is carried across the inner membrane via a monocarboxylic acid:proton symporter (Saier, 1987), and the ratio of pyruvate across the membrane is a direct function of $\Delta \mathrm{pH}$ or the hydrogen ion gradient across the inner mitochondrial membrane (LaNoue and Schoolwerth, 1979).

$\mathrm{CO}_{2}$ efflux must occur in reverse over the same pathway as does oxygen influx in order to move from the cells to a respiratory surface. $\mathrm{CO}_{2}$ diffuses about 20 times more rapidly than oxygen and hence partial pressure differences are far less than those for oxygen (Guyton, 1991). As a consequence, any change in tissue capillary blood flow will more dramatically affect $\mathrm{PCO}_{2}$ differences.

The significance of cardiovascular gas (oxygen and carbon dioxide) transport is further illustrated in experiments correlating muscle oxidative capability with mitochondrial and capillary density. In African airbreathing catfish, measurements were made of mitochondrial densities and capillary supply to Type I and Type II muscles. Type I fibres had a $15 \%$ fraction of fibre occupied by 
mitochondria as opposed to $2.5 \%$ for Type II fibres. Increased mitochondrial fraction was accompanied by a porportional increase in capillary density (Johnston and Bernard, 1983).

Similar results were obtained from studies involving Antarctic fishes (Londraville and Sidell, 1990). An additional observation was that in red, oxidative muscle, increases in mitochondrial and capillary density were associated with increased lipid density. In addition to acting as a fatty acid reservoir for mitochondrial oxidation, this lipid may serve to enhance oxygen flux to oxidative muscle since oxygen is four times more soluble in lipid than in aqueous solutions (Londraville and Sidell, 1990).

While cardiovascular limits may be dominant, other limiting factors can exist. Another potential source of limitation to $\mathrm{VO}_{2}$ max include enzymatic regulatory mechanisms and ATP/ADP ratios. Control of respiratory activity may be achieved by regulating the amount of enzyme made or by regulating the enzyme itself by product inhibition. ATP/ADP ratios have long been implicated in regulatory mechanisms (Tzagoloff, 1982). Studies performed with cardiac muscle mitochondria revealed a definite but unclear regulatory role of pyruvate dehydrogenase, alpha-ketoglutarate dehydrogenase, beta-hydroxybutyrate dehydrogenase, and branched-chain alphaketoacid dehydrogenase enzymes. Mitochondrial oxidation of glycolytic products, ketone bodies, and amino acid-derived 
substrates is achieved through optimal performance of the observed enzymes (Mela-Riker and Bukoski, 1985). Kunz et al. (1981) carried out a series of experiments which suggested that the rate of respiration responded to the extramitochondrial ATP/ADP ratio. This ratio is detected by the respiring mitochondria through the rate-limiting role of adenine translocase.

Increasing concentrations of $\mathrm{CO}_{2}$ and/or lactate may also influence substrate preference. Respiratory exchange ratios calculated for humans during exercise performed with varying inspired air $\mathrm{PCO}_{2}$ indicated an inhibition of glycolytic activity. This glycolytic inhibition was accompanied by a progressive shift towards lipid metabolism reflecting a shifted substrate preference in respiring mitochondria (Graham et al. 1982, 1980).

When isolated skeletal muscle mitochondria from both $\underline{B}$. marinus and $\underline{R}$. catesbeiana were polarographically monitored while oxidizing pyruvate, alpha-ketoglutarate, octanoylcarnitine, and oxaloacetate, no significant difference $(P<0.05)$ in rank-order preference was found. Both species preferred each of the five substrates in identical order. However, mitochondrial $\mathrm{VO}_{2} \max$ in $\underline{\mathrm{B}}$. marinus was significantly greater than that of $\underline{R}$. catesbeiana in all instances except when oxaloacetate was given as a substrate, confirming the generalization that oxaloacetate is a poor substrate for vertebrate mitochondria. Also, B. marinus 
yielded a higher number of mitochondria per gram of excised skeletal muscle.

In contrast, substrate preference in carp red and white muscle mitochondria (Moyes et al. 1989) demonstrated a profound difference. White muscle remained primarily glycolytic where mitochondria would expect pyruvate as a substrate once oxygen became available. Red muscle however, relied on a wider range of fuels, especially fatty acids, which would yield more ATP after complete mitochondrial oxidation.

Differences between carp and anurans may be explained by the fact that carp have histologically distinct red and white locomotory muscle groups while anurans do not. Anuran locomotory muscles are composed of three fibre types which occur in similar proportions regardless of the individual muscle or even species examined (Putnam and Bennett, 1983). Despite the results in relative substrate preference, behavioral and ecological correlates with other aspects of organismic metabolic capacity may occur. Mitochondrial density has been correlated with muscle fibre type and $\mathrm{VO}_{2}$ max for antarctic fish (Londraville and Sidell, 1990); mammalian quadrapeds (Hoppeler, 1990; Hoppeler et al. 1987); and rabbits (Pande and Blanchaer, 1971). The data collected for $\underline{B}$. marinus and $\underline{R}$. catesbeiana also agree with these previous observations which contend that red muscle is better suited for oxidative metabolism since a greater number of 
mitochondria exist. Thus it appears $\underline{B}$. $\underline{\text { marinus }}$ is overall more capable of sustained exercise as reflected in the higher yield of isolated mitochondria. Indeed, the enzymatic activity of citrate synthase was higher in $\underline{B}$. boreas and contractile speed of muscle groups slower when compared to $\underline{R}$. pipiens (Putnam and Bennett, 1983).

Although $\underline{B}$. marinus and $\underline{R}$. catesbeiana did not display any relative difference in substrate preference, the rank order in which both amphibians utilized the various substrates may characterize certain aspects of amphibian metabolism.

Isolated skeletal muscle mitochondria from $\underline{B}$. marinus and $\underline{R}$. catesbeiana exhibited maximal state 3 values with alpha-ketoglutarate as the substrate. This implies the existance of a tricarboxylate alpha-ketoglutarate/malate antiporter in amphibian skeletal muscle which is as significant a carrier as the expected monocarboxylate pyruvate $/ \mathrm{H}^{+}$symporter. It may also indicate a diet enriched with protein. This is in contrast to blowfly skeletal muscle mitochondria which maintain no tricarboxylate shuttle (Tzagoloff, 1982). It must also be considered that in cardiac muscle mitochondria, the presence of malate in the oxygen uptake media stimulated uptake of alpha-ketoglutarate (LaNoue et 21.1970 ) and the presence of malate in the oxygen uptake media of this procedure may be partially responsible for such high rates of uptake in the amphibian subjects. 
Oxaloacetate was a relatively poor substrate as reflected in low $\mathrm{RCR}$ and state 3 values. Oxaloacetate is transported via a dicarboxylate antiporter which exchanges malate, succinate, malonate, or oxaloacetate for phosphate. The efficiency of transport is greatest with malate and succinate, while oxaloacetate is transported poorly and only in the absence of other competing dicarboxylic acids (Tzagoloff, 1982).

Pyruvate was oxidized at rates $70 \%$ of alphaketoglutarate as was octanoyl-carnitine. In tissues that are less biosynthetically active, mitochondrial rates of pyruvate oxidation reflect the ability of the tissue to utilize carbohydrate. Typically, pyruvate is oxidized at rates as great as any other substrate in vertebrate heart and skeletal muscle: mammalian heart (Bremer, 1965); skeletal muscle in mammals (Pande and Blanchaer, 1971); turtles (Mersmann and Privitera, 1964); birds (Suarez et al. 1986); and teleosts (Moyes et al. 1989).

Unlike liver, heart and skeletal muscle mitochondria are specialized for oxidation of acetyl-CoA produced via betaoxidation of fatty acids. Red muscle mitochondria prefer long-chain fatty acid (LCFA) carnitines in birds (Suarez et al. 1986) and carp red muscle (Moyes et al. 1989). Red muscle muscle mitochondria also oxidize LCFA-carnitines at higher rates than do white muscle mitochondria (Moyes et al. 1989; Pande and Blanchaer, 1971). ‥ marinus and $\mathrm{R}$. 
catesbeiana both utilize octanoyl-carnitine at relatively high rates which indicates considerable aerobic capacity for both species.

Beta-hydroxybutyrate was consumed at $50 \%$ the rate alphaketoglutarate was for both $\underline{B}$. marinus and $\underline{R}$. catesbeiana. In response to low glucose levels and hormonal signals, increased lipolysis and levels of carnitine enhance fatty acid delivery to the liver. When free fatty-acid oxidation exceeds the capacity of the Kreb's cycle to oxidize acetylCoA, acetyl-CoA is used to synthesize ketone bodies: acetoacetate, beta-hydroxybutyrate, and acetone (Patton et a1. 1989).

The observed mitochondrial rate of beta-hydroxybutyrate metabolism in amphibians is consistant with other vertebrates in which extrahepatic ketone utilization depends on substrate availability (see Moyes, Suarez, and Hochachka, 1990). Betahydroxybutyrate is utilized at similar rates in elasmobranchs heart and red muscle mitochondria (Moyes, Buck, and Hochachka, 1990), in extrahepatic tissue in sturgeon (Singer et al. 1990), turtle (Mersmann and Privitera, 1964), and heart mitochondria from rat and pigeon (Tyler and Gonze, 1967). An exception among the vertebrates are teleosts, which oxidize ketone bodies at relatively low rates (Moyes et a․ 1989).

In mammals, ketone body utilization occurs when carbohydrate levels are attenuated and hepatic ketogenesis 
affords extrahepatic tissues fatty-acid derived substrate that may be used in the Kreb's cycle. Some workers have also hypothesized that fish red muscle has a function somewhat similar to that of the mammalian liver. Evidence for this idea includes similarities in the fat composition and vitamin content (Johnston et al. 1983). This hypothesis also includes a proposal that there is a non-circulatory transfer of metabolites between red and white muscles during exercise. In particular, the transfer of lactate from white to red muscle and the transfer of glucose from red to white muscle. Another possible advantage to ketone body metabolism in anuran amphibians centers around the homogeneous distribution of Type I and II skeletal muscle. During exercise, glycogen stores may quickly be exhausted. Ketone bodies provide a fatty-acid derived source of acetyl-CoA. For mixed muscle fibre tissues, this may be an optimal use of lipid material where intermittent mitochondria-rich red muscle fibre may effectively utilize the substrate.

Substrate preference in isolated Lake Charr liver mitochondria was shown to be temperature dependent (Ballantyne, Flannigan, and White, 1989). At low temperatures, the oxidation of ketone bodies increased to levels comparable to pyruvate or glutamate. Explanations include carbohydrate conservation or that enhanced oxidation of ketone bodies at low temperature serves to lower the 
availability of acetoacetate for the synthesis of cholesterol, which will stiffen cell membranes.

It now appears that substrate preference may be affected by temperature. For ectotherms, this is a significant factor to be considered for both interpretation of data as well as future experimental design.

The presented data on $\mathrm{pH}$ tolerance, taken in consideration with the available literature, supports the conclusion that $\mathrm{pH}$ balance is important for maximal mitochondrial oxidation, and the theory for carbon dioxide efflux as a potential limit to $\mathrm{VO}_{2} \max$. Also, substrate preference data has now been delineated for amphibians, the only vertebrate for which no data was available. Substrate preference data is consistant with that obtained for other vertebrates and supports a mixed muscle fibre type theory for anuran amphibians. It appears that the frog and toad are both adapted for aerobic metabolism as reflected by their identical substrate preference. However, the lower $\mathrm{VO}_{2} \max$ of the frog relative to the toad indicates the frog more frequently encounters the need for anaerobic muscle metabolism. 


\section{REFERENCES}

Ballantyne, J.S., D. Flannigan, and T.B. White. 1989. Effects of temperature on the oxidation of fatty acids, acylcarnitines, and ketone bodies by mitochondria from the liver of the lake charr, Salvelinus namacush. Canadian Journal of Fisheries and Aquatic Science. 46:950-954.

Bremer, J. 1965. The effect of acylcarnitines on the metabolism of pyruvate in rat heart mitochondria. Biochimica Et Biophysica Acta. 104:581-590.

Byrne, E. and I. Trounce. 1985. Oxygen electrode studies with Human skeletal muscle mitochondria in vitro. Journal of Neurological Sciences. 69:319-333.

Chance, B. and G.R. Williams. 1956. The respiratory chain and oxidative phosphorylation. Advances in Enzymology.17:65135 .

Cummings, J.W. 1979. Physiological and biochemical adaptations to training in Rana pipiens. Journal of Comparative Physiology 134:345-350.

DiPrampero, P.E. and G. Ferretti. 1990. Factors limiting maximal oxygen consumption in humans. Respiration Physiology. 80:113-128.

Estabrook, R.W. 1967. Mitochondrial respiratory control and the polarographic measurement of ADP:O ratios. Pages 4147. in: S.P. Colowick and N.O. Kaplan, eds. Methods in Enzymology vol 10. Academic Press, New York.

Garland, T. and R.B. Huey. 1987. Testing symmorphosis: Does structure match functional requirements? Evolution. 41:1404-1409.

Graham, T., B.A. Wilson, M. Sample, J. Van Dijk, and A. Bonen, 1980. The effects hypercapnia on metabolic responses to progressive exhaustive work. Medicine and Science in Sports and Exercise. 12:278-284.

Graham, T.E., B.A. Wilson, M. Sample, J. Van Dijk, and B. Goslin. 1982. The effects of hypercapnia on the metabolic response to steady-state exercise. Medicine and Science in Sports and Exercise. 14:286-291. 
Guyton, A.C. 1991. Textbook of Medical Physiology. 8th Ed. W.B. Saunders Company, Philadelphia.

Hillman, S.S. 1976. Cardiovascular correlates of maximal oxygen consumption rates in anuran amphibians. Journal of Comparative Physiology. 109:199-207.

Hillman, S.S. 1980. The effect of anemia on metabolic performance in the frog, Rana pipens. The Journal of Experimental Biology. 211:107-111.

Hillman, S.S. 1981. Effects of DL-propanolol on exercise heart rate and maximal rates of oxygen consumption in Scaphiopus intermentanus. Experientia 38:4-5.

Hillman, S.S. 1987. Dehydrational effects on cardiovascular and metabolic capacity in two amphibians. Physiological Zoology. 60:608-613.

Hillman, S.S., M.S. Lea, and J.M. Duerr. 1991. Osmotic effectson mitochondria from two species of amphibian, Bufo marinus and Rana catesbeiana. In Press, Physiological Zoology.

Hooker, A.M. and K.M. Baldwin. 1979. Substrate specificity in different types of mammalian muscle. American Journal of Physiology. 236:C66-C69.

Hoppeler, H., S.R. Kayar, H. Claassen, E. Uhlmann, and R.H. Karas. 1987. Adaptive variation in the mammalian respiratory system in relation to energetic demand: III. Skeletal muscles:setting the demand for oxygen. Respiration Physiology. 69:27-46.

Hoppeler, H. 1990. The different relationship of $\mathrm{VO}_{2}$ max to muscle mitochondria in humans and quadrupedal animals. Respiration Physiology. 80:137-146.

Johnston, I.A. 1977. Energy metabolism of carp swimming muscles. Journal of Comparative Physiology. 114:203-216.

Johnston, I.A. and L.M. Bernard. 1983. Aquatic and aerial respiration rates, muscle capillary supply and mitochondrial volume density in the air-breathing catfish (Clarias mossambicus) acclimated to either aerated or hypoxic water. Journal of Experimental Biology. 105:317-338. 
Kunz, W., R. Bohnensack, G. Bohne, U. Kuster, G. Letko, and P. Schonfeld. 1981. Relations between extramitochondrial and intramitochondrial adenine nucleotide systems.

Archives of Biochemistry and Biophysics. 209:219-229.

LaNoue, K., W.J. Nicklas, and J.R. Williamson. 1970. Control of citric acid cycle activity in rat heart mitochondria. Journal of Biological Chemistry. 245:102-111.

LaNoue, K.F., and A.C. Schoolwerth. 1979. Metabolite transport in mitochondria. Annual Review of Biochemistry. 48:871-922.

Lea, M.S. and S.S. Hillman. 1990. Effects of osmolality and solutes on performance of shark heart mitochondria. The Journal of Experimental Zoology. 255:9-15.

Londraville, R.L. and B.D. Sidell. 1990. Ultrastructure of aerobic muscle in antarctic fishes may contribute to maintainance of diffusive fluxes. Journal of Experimental Biology. 150:205- 220 .

Lowry, O.H., N.J. Rosebrough, A.L. Farr and R.J. Randall. 1951. Protein measurement with the Folin phenol reagent. Journal of Biological Chemistry. 193:265-275.

Moyes, C.D., L.T. Buck, P.W. Hochachka, and R.K. Suarez . 1989. Oxidative properties of carp red and white muscle. Journal of Experimental Biology. 143:321-331.

Moyes, C.D., R.K. Suarez, and P.W. Hochachka. 1989. A comparison of fuel preferences of mitochondria from vertebrates and invertebrates. Canadian Journal of Zoology. 68:1337-1349.

Moyes, C.D., L.T. Buck, and P.W. Hochachka. 1990. Mitochondrial and peroxisomal fatty acid oxidation in elasmobranchs. American Journal of Physiology. 258:R756R762.

Mela-Riker, L.M. and R.D. Bukoski. 1985. Regulation of mitochondrial activity in cardiac cells. Annual Reviews in Physiology. 47:645-663.

Mersmann, H.J., and C.A. Privitera. 1964. In vitro metabolism by turtle heart mitochondria. American Journal of Physiology. 206:980-984.

Pande, S.V. and M.C. Blanchaer. 1971. Carbohydrate and fat in energy metabolism of red and white muscle. American Journal of Physiology. 220:549-553. 
Patton, H.D., A.F. Fuchs, B. Hille, A.M. Scher, and R.Steiner Eds. 1989. Textbook of Physiology. 21st ed. W.B. Suanders and Company, Philadelphia.

Pearson, M.P., L.L. Spriet, and E.D. Stevens. 1990. Effect of sprint training on swim perfromance and white muscle metabolism during exercise and recovery in Rainbow trout (Salmo gairdneri). Journal of Experimental Biology. $149: 45-60$.

Putnam, R.W. 1979. The role of lactic acid accumulation in muscle fatigue of two species of anurans, Xenopus laevis and Bana pipiens. Journal of Experimental Biology. $82: 32-51$.

Putnam, R.W. and A.F. Bennett. 1983. Histochemical, enzymatic, and contractile properties of skeletal muscles of three anuran amphibians. American Journal of Physiology. $244:$ R558-R67.

Rayner, M.D. and M.J. Keenan. 1967. Role of red and white muscles in the swimming of skipjack tuna. Nature. $214: 392-393$.

Saier, M.H. 1987. Enzymes in Metabolic Pathways. Harper and Row, San Francisco.

Schmidt-Nielson, K. 1984. Animal Physiology. 3rd ed. Cambridge University Press, New York.

Singer, T.D., V.G. Mahadevappa and J.S. Ballantyne. 1990. Aspects of the energy metabolism of Lake Sturgeon, Acipenser fulvescens, with special emphasis on lipid and ketone body metabolism. Canadian Journal of Fisheries and Aquatic Science. 47:873-881.

Stryer, L. 1988. Biochemistry. 3rd ed.W.H. Freeman, New York.

Suarez, R.K., G.S. Brown and P.W. Hochachka. 1986. Metabolic sources of fuel for hummingbird flight. American Journal of Physiology. 251:R537-R542.

Taylor, C.R. and E.R. Weibel. 1981. Design of mammalian respiratory system I. Problem and strategy. Respiratory Physiology. 44:1-10.

Thorson, T.B. 1955, The relationship of water economy to terrestrialism in amphibians with their ability to survive loss of body water. Ecology. 24:374-381. 
Tyler, D.D. and J. Gonze. 1967. The preparation of heart mitochondria from laboratory animals. Methods in Enzymology. 10:75-77.

Tzagoloff, A. 1982. Mitochondria. Plemun Press, New York.

Withers, P.C. and S.S. Hillman. 1988. A steady-state model of maximal oxygen and carbon dioxide transport in anuran amphibians. Journal of Applied Physiology. 64:860-868.

Walsberg, G.E., M.S. Lea, and S.S. Hillman. 1986. Individual variation in maximum aerobic capacity: cardiovascular and enzymatic correlates in Rana catesbeiana. The Journal of Experimental Zoology. 239:1-5.

Zubay, G. 1988. Biochemistry. 2nd ed. Macmillan Publishing Company, New York. 